\title{
Distancia a la frontera e impacto asimétrico en los sectores económicos por las políticas de desregulación*
}

\author{
Gonzalo Jiménez Jaramillo*", Conrado Giraldo Zuluaga***
}

\begin{abstract}
Resumen
Introducción. En este artículo se analiza el impacto de las políticas de desregulación propuestas por el Banco Mundial (BM) con el fin de que las economías puedan mejorar su capacidad de hacer negocios en los sectores de comercio de bienes y de comercio de servicios. Objetivo. El propósito es identificar el impacto asimétrico en los principales sectores y las consecuencias económicas en la reducción de la pobreza debido a la forma como están diseñadas las políticas de desregulación. Materiales y métodos. Mediante el uso de herramientas estadísticas se han efectuado análisis de correlación en las mediciones históricas que el BM ha recopilado durante el período 2010-2012. Resultados. Se encontró que las políticas de desregulación producen un efecto distinto en el comercio de mercancías y en el comercio de bienes intangibles. Conclusión. El análisis de los resultados deja en evidencia que dichas políticas inducen a los países a tener un mejor desempeño en el comercio de mercancías y no en el comercio de servicios. Lo anterior resulta catastrófico para el propósito de reducir la pobreza, debido a que el comercio de intangibles es el que genera una mayor riqueza, mientras que el comercio de mercancías da como resultado ganancias marginales y rezago económico.
\end{abstract}

Palabras clave: distancia a la frontera, comercio de mercancías, comercio de servicios, desregulación, desempeño económico.

\author{
Distance to the border and asymmetric \\ impact on the economic sectors due to \\ deregulation policies
}

\begin{abstract}
Introduction. In this article the impact of the deregulation policies proposed by the World Bank in order to increase the economies' capacity to make businesses in the trade of goods and services are analyzed. Objective. To identify the asymmetric impact on the main sectors and the economic consequences on the reduction of poverty due to the way the deregulation policies are designed. Materials and methods. By the use of statistic tools, correlation analyses have been performed on the historical measurements compiled by the World Bank in the period between the years 2010-2012. Results. The deregulation policies have a different effect on the trade of goods, in comparison to the trade of services. Conclusion. The analysis of the results shows that such policies help countries to have a better performance in the trade of goods, but not in that of services. This is catastrophic for the goal of reducing poverty, due to the fact that the trade of services generates more wealth, while the trade of goods produces marginal gains and economic backwardness.
\end{abstract}

Key words: distance to border, trade of godos, trade of services, deregulation, economic performance.

\footnotetext{
* Artículo original de investigación de la línea "Postmodernidad y Revolución de la Información" dentro de las actividades de los grupos de investigación "Filosofía y Teología Crítica" y "GORAS" de la Fundación Universitaria Luis Amigó en Medellín-Colombia y financiado por la misma universidad en el período comprendido 2014-2015.

** Doctor en Filosofía por la Universidad Pontificia Bolivariana (Medellín-Colombia), magíster en Administración e Ingeniero de Producción de la Universidad EAFIT (Medellín-Colombia). Docente investigador de la Fundación Universitaria Luis Amigó de Medellín

*** Doctor en Filosofía, magíster en Desarrollo y filósofo por la Universidad Pontificia Bolivariana (Medellín-Colombia). Profesor titular de la Facultad de Filosofía de esta Universidad. Hace parte del Grupo de Investigación Epimeleia. Colabora como articulista en varias revistas nacionales y foráneas.
} 


\section{Distância à fronteira e impacto assimétrico nos setores económicos pelas políticas de desregulação}

\section{Resumo}

Introdução. Neste artigo se analisa o impacto das políticas de desregulação propostas pelo Banco Mundial (BM) com o fim de que as economias possam melhorar sua capacidade de fazer negócios nos setores de comércio de bens e de comércio de serviços. Objetivo. O propósito é identificar o impacto assimétrico nos principais setores e as consequências económicas na redução da pobreza devido à forma como estão desenhadas as políticas de desregulação. Materiais e métodos. Mediante o uso de ferramentas estatísticas se efeituaram análise de correlação nas medições históricas que o BM há recopilado durante o período 20102012. Resultados. Se encontrou que as políticas de desregulação produzem um efeito diferente no comércio de mercancias e no comércio de bens intangíveis. Conclusão. A análise dos resultados deixa em evidência que ditas políticas induzem aos países a ter um melhor desempenho no comércio de mercancias e não no comercio de serviços. O anterior resulta catastrófico para o propósito de reduzir a pobreza, devido a que o comércio de intangíveis é o que gera uma maior riqueza, enquanto que o comercio de mercancias dá como resultado lucros marginais e deficiência económica.

Palavras chave: distância à fronteira, comércio de mercancias, comercio de serviços, desregulação, desempenho económico.

\section{Introducción}

El Banco Mundial (BM) ha desarrollado un indicador llamado Distancia a la Frontera (DF) que, a partir del año 2005, muestra empíricamente el nivel absoluto de desempeño en materia regulatoria de las economías, con el fin de conocer la forma en que cambian a través del tiempo a medida que los gobiernos implementan acciones orientadas a la desregulación para incrementar la facilidad en los negocios desde distintos aspectos: Global excluyendo obtención de electricidad, Apertura de un negocio, Manejo de permisos de construcción, Obtención de electricidad, Registro de propiedades, Obtención de crédito, Protección de los inversionistas minoritarios, Pago de impuestos, Comercio transfronterizo, Cumplimiento de contratos y Resolución de la insolvencia.

El indicador Distancia a la Frontera (DF) también evalúa el cambio absoluto en el entorno regulatorio de una economía a través del tiempo. Se presenta en una escala de 0 a 100 , siendo 0 el valor más bajo de la escala, y 100 , el más alto y óptimo, el cual se conoce como frontera. El BM publica un informe anual llamado "Doing Business" en el cual se presenta la Distancia a la Frontera de la gran mayoría de las economías del mundo.

En la medida en que los países implementan acciones de desregulación en el entorno de los negocios, se espera que cada año se acerquen más a la frontera. La evidencia de los resultados y las metodologías utilizadas por el BM para la elaboración del instrumento de medición (Banco Mundial, 2014) no se objetan en el presente artículo.

Sin embargo, existe la incertidumbre de la forma como estas acciones de desregulación están orientadas a los diferentes sectores de la economía; específicamente a los sectores de comercio de servicios y comercio de mercaderías. Se tiene el interrogante sobre la posibilidad de efectos desiguales que en ambos sectores ejercen las políticas de desregulación que incentiva el BM para que las economías exhiban un mejor desempeño.

Las dudas surgen a partir de la forma como está configurado el marco de acciones, el cual busca reducir las problemáticas derivadas de la gestión de instalaciones físicas, la movilización transfronteriza, la obtención de electricidad o el crédito financiero, los cuales son prioritarios en materia de producción industrial y comercialización de sus productos representados en bienes materiales, pero no resultan críticos en la producción de conocimiento y en la comercialización de sus productos intangibles constituidos en gran medida bajo la figura del servicio.

Dada la naturaleza de los aspectos mencionados arriba, se plantea la hipótesis de 
que la desregulación, cuya eficiencia mide el $\mathrm{BM}$ a través de la DF, tiene un mayor impacto en el comercio de mercaderías que en el de servicios.

Esto, más allá que exponer las características de medición del indicador DF, pretende poner en relieve aspectos más profundos derivados de la naturaleza de este parámetro en la medida en que condiciona y compromete a las economías, en la búsqueda de la eficiencia según el modelo del BM, con el sector de mercaderías incentivando con ello la producción industrial y rezagando los aspectos que promueve el comercio de servicios, y que son inherentes al desarrollo y al bienestar, como la producción intelectual.

Elanálisis de esteaspecto es de suma relevancia en el contexto de una sociedad posindustrial, economías digitales, o sociedad de información, en las que los niveles de rentabilidad del capital relacionado con la producción de mercaderías frente a los de la producción de intangibles ${ }^{1}$, lo que el BM llama "servicios", empiezan a tener una proporcionalidad que determina la competitividad y riqueza de la economía a partir de sus compromisos o bien con las actividades de producción industrial o con el negocio de lo inmaterial derivada de la producción del saber:

En su conjunto la productividad del saber va a ser cada vez más el factor determinante en la posición competitiva de un país, una industria, una empresa. Respecto del saber, ningún país, ninguna empresa, tiene ventajas o desventajas "naturales". La única ventaja que puede tener es respecto de cuánto obtiene del saber disponible para todos. Lo único que importará cada vez más en la economía nacional e internacional serán los resultados que consiga en productividad del saber (Drucker, 1993, 237).

En este sentido cobra fuerza la idea del comercio de servicios como una actividad que da cuenta del grado de inserción de una economía en la actividad intelectual y se hace indispensable la revisión de aquellos elementos que pueden en resultar inocuos, pero que llevan a inconsistencias en las pretensiones de mejorar la calidad de vida y el futuro de los individuos.

\section{Materiales y métodos}

Las variables de análisis del estudio son: Distancia a la Frontera ${ }^{2}$ (DF), Comercio de Servicios como porción del $\mathrm{PIB}^{3}$ (SERV) y Comercio de Mercaderías como porción del PIB (MERC). Estas variables fueron publicadas por el Banco Mundial (BM), y para el presente estudio fueron restringidas al período del 2010 a 2012 y únicamente para 141 economías (tablas 1, 2 y 3 del anexo) de las 268 definidas hasta el año 2014 (Banco Mundial G. , Doing Business. Midiendo regulaciones para hacer negocios, 2014). Se incluyeron solo aquellas economías para las que se dispone de la mayor cantidad de datos completos y consecutivos a la fecha ${ }^{4}$.

1 Al respecto, Plata (2005) dice: “... en general los denominados bienes intangibles están dotados, gracias a las actuales condiciones de la economía, de unas características especiales, que los convierten en bienes de capital autónomos del soporte material de las grandes instalaciones de concreto o de las grandes inversiones en activos tangibles, indispensables en los procesos de producción y, por tanto, inmersos en las reglas del mercado, susceptibles de apreciarse o depreciarse según las reglas de oferta y demanda y capaces de obtener un mercado propio".

2 El indicador de eficiencia "Distancia a la Frontera" utilizado para este caso ha sido únicamente el "Global excluyendo electricidad" dado que de todos los posibles que desarrolla el BM es el que abarca la mayor cantidad de acciones de desregulación (Banco Mundial G. Doing Business. Midiendo regulaciones para hacer negocios, 2014).

3 Producto Interno Bruto.

4 Información encontrada en las bases de datos del Banco Mundial a Noviembre 15 de 2014 
Tabla 1. Distancia a la Frontera (DF)

\begin{tabular}{|c|c|c|c|}
\hline \multirow{2}{*}{ Economía } & DF & DF & DF \\
\hline & Doing Business 2010 & Doing Business 2011 & Doing Business 2012 \\
\hline Afganistán & 40,98 & 38,25 & 39,74 \\
\hline Albania & 62,68 & 62,88 & 59,21 \\
\hline Alemania & 79,96 & 79,92 & 80,06 \\
\hline Angola & 37,73 & 37,61 & 40,63 \\
\hline Antigua y Barbuda & 65,39 & 65,24 & 64,94 \\
\hline Arabia Saudita & 72,17 & 73,71 & 73,17 \\
\hline Argelia & 52,15 & 51,88 & 52,08 \\
\hline Argentina & 56,9 & 56,76 & 57,19 \\
\hline Armenia & 61,25 & 61,8 & 64,3 \\
\hline Australia & 80,87 & 81,15 & 80,99 \\
\hline Austria & 76,69 & 77,05 & 77,05 \\
\hline Azerbaiyán & 62,53 & 62,59 & 63,61 \\
\hline Bahamas & 65,67 & 65,69 & 65,25 \\
\hline Bahrein & 67,41 & 67,53 & 67,59 \\
\hline Bangladesh & 47,43 & 49,25 & 48 \\
\hline Belarús & 54,36 & 56,86 & 62,02 \\
\hline Bélgica & 74,08 & 73,83 & 74,08 \\
\hline Belice & 61,05 & 60,94 & 61,5 \\
\hline Bhután & 55,06 & 54,46 & 57,32 \\
\hline Bolivia & 49,51 & 49,75 & 50,09 \\
\hline Bosnia y Herzegovina & 52,95 & 54,14 & 55,57 \\
\hline Botswana & 64,72 & 65,15 & 65,71 \\
\hline Brasil & 53,57 & 53,21 & 53,16 \\
\hline Bulgaria & 68,45 & 68,58 & 68,26 \\
\hline Burundi & 35,7 & 35,89 & 41,62 \\
\hline Cabo Verde & 53,32 & 55,82 & 58,58 \\
\hline Camboya & 48,4 & 49,66 & 50,18 \\
\hline Camerún & 43,33 & 45,38 & 47,75 \\
\hline Canadá & 79,98 & 80,97 & 80,56 \\
\hline Chile & 68,12 & 69,52 & 72,06 \\
\hline China & 57,79 & 59,73 & 58,78 \\
\hline Chipre & 66,27 & 67,5 & 68,86 \\
\hline Colombia & 65,7 & 66,85 & 69,26 \\
\hline $\begin{array}{c}\text { Congo, República } \\
\text { Democrática }\end{array}$ & 31,49 & 34,66 & 35,74 \\
\hline Corea, República de & 81,74 & 81,98 & 82,9 \\
\hline Costa Rica & 57,44 & 57,14 & 58,37 \\
\hline Croacia & 60,92 & 61,1 & 62,32 \\
\hline Dinamarca & 84,24 & 85,42 & 85,72 \\
\hline Djibouti & 44,11 & 43,4 & 43,13 \\
\hline Dominica & 63,97 & 63,88 & 64,06 \\
\hline Ecuador & 57,12 & 57,19 & 57,54 \\
\hline Egipto & 56,07 & 57 & 58,04 \\
\hline El Salvador & 59,74 & 59,18 & 59,34 \\
\hline Eslovaquia & 68,88 & 70,17 & 70,17 \\
\hline Eslovenia & 63,43 & 66,32 & 68,13 \\
\hline España & 70,75 & 70,82 & 72,73 \\
\hline Estados Unidos & 85,72 & 85,72 & 85,65 \\
\hline
\end{tabular}




\section{Continuación tabla 1}

\begin{tabular}{|c|c|c|c|}
\hline \multirow{2}{*}{ Economía } & DF & DF & DF \\
\hline & Doing Business 2010 & Doing Business 2011 & Doing Business 2012 \\
\hline Estonia & 75,82 & 76,18 & 75,82 \\
\hline Etiopía & 48,4 & 50,35 & 50,92 \\
\hline Fiji & 68,19 & 68,45 & 68,33 \\
\hline Filipinas & 54,31 & 55,02 & 55,68 \\
\hline Finlandia & 81 & 81,55 & 82,39 \\
\hline Francia & 70,42 & 71,09 & 71,12 \\
\hline Gambia & 48,47 & 48,77 & 49,9 \\
\hline Georgia & 74,3 & 76,74 & 79,3 \\
\hline Ghana & 61,47 & 63,61 & 64,28 \\
\hline Grenada & 59,22 & 60,29 & 60,31 \\
\hline Grecia & 62,15 & 60,13 & 60,62 \\
\hline Guatemala & 61,3 & 61,25 & 61,67 \\
\hline Guinea & 37,23 & 35,97 & 38,59 \\
\hline Guyana & 58,37 & 58,6 & 58,44 \\
\hline Haití & 42,99 & 43,12 & 42,45 \\
\hline Honduras & 59,28 & 58,41 & 59,33 \\
\hline Hong Kong RAE, China & 88,95 & 89,34 & 90,01 \\
\hline Hungría & 65,32 & 67,15 & 66,89 \\
\hline India & 49,46 & 50,76 & 53,08 \\
\hline Indonesia & 57,18 & 57,76 & 58,47 \\
\hline Iraq & 50,86 & 50,18 & 50,5 \\
\hline Irlanda & 82,27 & 81,69 & 81,15 \\
\hline Islandia & 80,74 & 81,07 & 82,33 \\
\hline Islas Salomón & 55,08 & 59,57 & 60,95 \\
\hline Israel & 71,29 & 71,81 & 71,79 \\
\hline Italia & 65,63 & 66 & 66,34 \\
\hline Jamaica & 62,54 & 63,54 & 63,95 \\
\hline Japón & 78,42 & 78,01 & 78,31 \\
\hline Jordania & 57,01 & 57,44 & 58,26 \\
\hline Kazajstán & 57,07 & 60,24 & 63,18 \\
\hline Kenia & 56,57 & 56,82 & 56,95 \\
\hline Kuwait & 61,85 & 61,67 & 62,81 \\
\hline Lesotho & 50,57 & 51,64 & 51,97 \\
\hline Líbano & 61,98 & 62,3 & 62,7 \\
\hline Lituania & 73,97 & 74,01 & 74,79 \\
\hline Luxemburgo & 65,5 & 65,81 & 66,74 \\
\hline Malasia & 74,37 & 76,19 & 79,63 \\
\hline Malawi & 48,32 & 49,2 & 49,6 \\
\hline Maldivas & 62,01 & 66,3 & 66,52 \\
\hline Marruecos & 60,86 & 60,28 & 63,61 \\
\hline Mauricio & 73,62 & 74,14 & 74,21 \\
\hline México & 65,1 & 67,44 & 69,04 \\
\hline Mongolia & 59,1 & 59,17 & 60,38 \\
\hline Montenegro & 63,74 & 64,7 & 66,14 \\
\hline Mozambique & 51,24 & 52,62 & 51,3 \\
\hline Nepal & 60,43 & 58,13 & 59,56 \\
\hline Nicaragua & 54,35 & 54,46 & 57,44 \\
\hline Nigeria & 42,83 & 42,65 & 43,93 \\
\hline
\end{tabular}




\section{Continuación tabla 1}

\begin{tabular}{|c|c|c|c|}
\hline \multirow{2}{*}{ Economía } & DF & DF & DF \\
\hline & Doing Business 2010 & Doing Business 2011 & Doing Business 2012 \\
\hline Noruega & 82,4 & 82,52 & 82,55 \\
\hline Nueva Zelandia & 88,83 & 89,07 & 89,09 \\
\hline Omán & 65,98 & 66,02 & 67,75 \\
\hline Países Bajos & 75 & 74,99 & 75,66 \\
\hline Pakistán & 56,35 & 56,24 & 57,29 \\
\hline Panamá & 65,51 & 66,03 & 66,13 \\
\hline Papua Nueva Guinea & 57,29 & 57,1 & 57,24 \\
\hline Paraguay & 60,47 & 61,2 & 61,5 \\
\hline Perú & 67,2 & 68,87 & 70,13 \\
\hline Polonia & 62,35 & 64,38 & 65,18 \\
\hline Portugal & 71,42 & 73,84 & 74,8 \\
\hline Reino Unido & 83,4 & 84,19 & 84,32 \\
\hline República Checa & 62,56 & 67,11 & 67,64 \\
\hline República Dominicana & 62,74 & 62,37 & 62,07 \\
\hline Rumania & 64,14 & 63,92 & 63,94 \\
\hline $\begin{array}{c}\text { Rusia, Federación } \\
\text { de - Moscú }\end{array}$ & 54,84 & 54,25 & 56,65 \\
\hline Rwanda & 58,33 & 60,06 & 60,27 \\
\hline Samoa & 65,27 & 67,24 & 67,5 \\
\hline $\begin{array}{c}\text { San Vicente y las } \\
\text { Granadinas }\end{array}$ & 63,59 & 63,77 & 63,91 \\
\hline Santa Lucía & 65,66 & 65,43 & 65,41 \\
\hline Santo Tomé y Príncipe & 43,51 & 41,68 & 48,49 \\
\hline Senegal & 41,89 & 42,08 & 43,94 \\
\hline Serbia & 59 & 59,72 & 60,4 \\
\hline Seychelles & 62,58 & 62,49 & 63,12 \\
\hline Sierra Leona & 39,87 & 44,31 & 48,39 \\
\hline Singapur & 91,85 & 92,48 & 92,48 \\
\hline Sri Lanka & 57,07 & 56,04 & 57,12 \\
\hline Sudáfrica & 69,09 & 69,47 & 70,55 \\
\hline Sudán & 49,27 & 49,43 & 49,86 \\
\hline Suecia & 81,78 & 83,26 & 82,84 \\
\hline Suiza & 75,73 & 75,78 & 75,86 \\
\hline Suriname & 43,76 & 44,34 & 44,5 \\
\hline Swazilandia & 54,75 & 58,23 & 58,64 \\
\hline Tailandia & 76,43 & 75,87 & 75,81 \\
\hline Tanzania & 52,62 & 55,38 & 56,51 \\
\hline Tayikistán & 40,5 & 44,18 & 44,42 \\
\hline Timor-Leste & 41,96 & 42,58 & 42,95 \\
\hline Tonga & 64,58 & 64,69 & 65,74 \\
\hline Túnez & 66,89 & 68,69 & 68,78 \\
\hline Turquía & 65,97 & 66,02 & 66,71 \\
\hline Ucrania & 40,42 & 44,73 & 45 \\
\hline Uganda & 48,94 & 51,14 & 50,05 \\
\hline Uruguay & 58,98 & 58,93 & 62,01 \\
\hline Vanuatu & 61,29 & 61,63 & 62,84 \\
\hline Venezuela, RB & 36,72 & 38,35 & 38,03 \\
\hline Viet Nam & 59,44 & 60,24 & 60,31 \\
\hline Zambia & 57,57 & 57,28 & 57,35 \\
\hline
\end{tabular}

Fuente: Doing Business, Banco Mundial 
Tabla 2. Comercio de servicios como porción del PIB (SERV)

\begin{tabular}{|c|c|c|c|}
\hline Economía & SERV 2010 & SERV 2011 & SERV 2012 \\
\hline Afganistán & 27,61 & 26,67 & 25,85 \\
\hline Albania & 38,52 & 39,27 & 34,69 \\
\hline Alemania & 16,03 & 16,01 & 16,69 \\
\hline Angola & 23,78 & 23,44 & 19,85 \\
\hline Antigua y Barbuda & 61,92 & 61,40 & 59,32 \\
\hline Arabia Saudita & 16,60 & 13,37 & 11,51 \\
\hline Argelia & 9,66 & 8,19 & 7,38 \\
\hline Argentina & 6,07 & 5,93 & 5,52 \\
\hline Armenia & 19,08 & 21,20 & 21,31 \\
\hline Australia & 8,59 & 8,19 & 7,68 \\
\hline Austria & 24,68 & 25,14 & 26,14 \\
\hline Azerbaiyán & 12,14 & 13,47 & 17,81 \\
\hline Bahamas & 46,59 & 48,09 & 51,90 \\
\hline Bahrein & 23,87 & 17,47 & 15,04 \\
\hline Bangladesh & 6,81 & 6,90 & 7,12 \\
\hline Belarús & 14,13 & 15,00 & 15,92 \\
\hline Bélgica & 37,51 & 36,95 & 40,78 \\
\hline Belice & 36,91 & 34,34 & 37,81 \\
\hline Bhután & 13,19 & 14,04 & 16,07 \\
\hline Bolivia & 9,45 & 10,85 & 11,38 \\
\hline Bosnia y Herzegovina & 15,11 & 14,50 & 14,34 \\
\hline Botswana & 7,32 & 8,99 & 6,46 \\
\hline Brasil & 4,39 & 4,62 & 5,37 \\
\hline Bulgaria & 22,42 & 21,90 & 22,76 \\
\hline Burundi & 12,23 & 13,78 & 12,31 \\
\hline Cabo Verde & 48,97 & 49,28 & 52,29 \\
\hline Camboya & 23,49 & 27,56 & 29,11 \\
\hline Camerún & 13,44 & 14,94 & 14,14 \\
\hline Canadá & 10,85 & 10,82 & 10,57 \\
\hline Chile & 10,95 & 11,48 & 10,40 \\
\hline China & 6,16 & 5,79 & 5,81 \\
\hline Chipre & 45,41 & 47,18 & 47,83 \\
\hline Colombia & 4,21 & 4,18 & 4,26 \\
\hline Congo, República Democrática del & 14,87 & 15,23 & 9,39 \\
\hline Corea, República de & 16,74 & 16,33 & 17,91 \\
\hline Costa Rica & 21,55 & 20,16 & 20,51 \\
\hline Croacia & 25,85 & 27,14 & 28,17 \\
\hline Dinamarca & 36,27 & 37,50 & 39,40 \\
\hline Djibouti & 38,29 & 37,64 & 35,11 \\
\hline Dominica & 43,33 & 43,98 & 43,51 \\
\hline Ecuador & 6,64 & 6,19 & 6,00 \\
\hline Egipto, República Árabe de & 17,60 & 14,07 & 14,54 \\
\hline El Salvador & 12,13 & 12,20 & 12,97 \\
\hline República Eslovaca & 14,50 & 14,21 & 14,99 \\
\hline Eslovenia & 21,92 & 22,48 & 23,90 \\
\hline España & 15,31 & 16,42 & 17,16 \\
\hline Estados Unidos & 6,44 & 6,76 & 6,74 \\
\hline Estonia & 39,14 & 42,09 & 42,77 \\
\hline Etiopía & 16,03 & 19,47 & 14,76 \\
\hline
\end{tabular}




\section{Continuación tabla 2}

\begin{tabular}{|c|c|c|c|}
\hline Economía & SERV 2010 & SERV 2011 & SERV 2012 \\
\hline Fiji & 44,75 & 45,50 & 44,52 \\
\hline Filipinas & 13,44 & 13,58 & 13,31 \\
\hline Finlandia & 20,57 & 21,02 & 22,16 \\
\hline Francia & 13,89 & 14,91 & 14,38 \\
\hline Gambia & 21,41 & 23,62 & 25,35 \\
\hline Georgia & 23,49 & 22,75 & 25,30 \\
\hline Ghana & 13,93 & 14,00 & 17,96 \\
\hline Granada & 31,95 & 33,27 & 31,20 \\
\hline Grecia & 19,73 & 20,63 & 20,69 \\
\hline Guatemala & 11,30 & 9,76 & 9,73 \\
\hline Guinea & 9,81 & 12,90 & 18,60 \\
\hline Guyana & 26,20 & 28,38 & 28,70 \\
\hline Haití & 22,90 & 18,48 & 18,09 \\
\hline Honduras & 20,69 & 20,90 & 20,32 \\
\hline $\begin{array}{l}\text { Hong Kong, } \\
\text { Región Administrativa Especial }\end{array}$ & 66,04 & 66,70 & 65,17 \\
\hline Hungría & 26,51 & 29,07 & 30,78 \\
\hline India & 13,57 & 14,02 & 14,80 \\
\hline Indonesia & 6,16 & 6,31 & 6,63 \\
\hline Iraq & 8,89 & 7,29 & 7,47 \\
\hline Irlanda & 93,80 & 97,24 & 103,97 \\
\hline Islandia & 37,52 & 39,54 & 42,72 \\
\hline Islas Salomón & 43,13 & 38,31 & 34,71 \\
\hline Israel & 18,79 & 18,57 & 20,15 \\
\hline Italia & 10,11 & 10,14 & 10,45 \\
\hline Jamaica & 33,70 & 31,64 & 31,83 \\
\hline Japón & 5,39 & 5,30 & 5,37 \\
\hline Jordania & 37,91 & 33,34 & 32,98 \\
\hline Kazajstán & 10,44 & 8,14 & 8,69 \\
\hline Kenya & 18,07 & 18,36 & 18,15 \\
\hline Kuwait & 20,64 & 17,22 & 16,14 \\
\hline Lesotho & 22,73 & 21,24 & 22,50 \\
\hline Libano & 76,49 & 81,43 & 79,94 \\
\hline Lituania & 23,54 & 21,54 & 26,93 \\
\hline Luxemburgo & 194,42 & 197,15 & 207,85 \\
\hline Malasia & 26,05 & 25,69 & 26,33 \\
\hline Malawi & 4,69 & 4,78 & 7,99 \\
\hline Maldivas & 105,96 & 117,76 & 123,16 \\
\hline Marruecos & 24,36 & 24,67 & 24,47 \\
\hline Mauricio & 48,09 & 51,33 & 51,10 \\
\hline México & 3,90 & 3,93 & 3,95 \\
\hline Mongolia & 20,56 & 27,46 & 29,34 \\
\hline Montenegro & 36,55 & 38,28 & 41,03 \\
\hline Mozambique & 19,95 & 23,06 & 36,67 \\
\hline Nepal & 9,65 & 8,73 & 9,48 \\
\hline Nicaragua & 15,83 & 16,30 & 16,62 \\
\hline Nigeria & 6,69 & 6,76 & 5,76 \\
\hline Noruega & 20,55 & 18,07 & 17,48 \\
\hline Nueva Zelandia & 14,94 & 15,16 & 14,76 \\
\hline
\end{tabular}




\section{Continuación tabla 2}

\begin{tabular}{|c|c|c|c|}
\hline Economía & SERV 2010 & SERV 2011 & SERV 2012 \\
\hline Omán & 13,93 & 13,17 & 14,76 \\
\hline Países Bajos & 23,53 & 24,30 & 25,94 \\
\hline Pakistán & 7,76 & 6,11 & 6,71 \\
\hline Panamá & 31,93 & 36,61 & 35,55 \\
\hline Papua Nueva Guinea & 32,35 & 27,35 & 26,89 \\
\hline Paraguay & 7,38 & 6,85 & 6,69 \\
\hline Perú & 6,27 & 5,96 & 6,12 \\
\hline Polonia & 13,27 & 13,47 & 14,24 \\
\hline Portugal & 15,83 & 17,18 & 17,86 \\
\hline Reino Unido & 18,52 & 19,29 & 19,22 \\
\hline República Checa & 19,22 & 20,02 & 21,85 \\
\hline República Dominicana & 17,84 & 17,33 & 17,39 \\
\hline Rumania & 11,12 & 11,78 & 12,94 \\
\hline Federación de Rusia & 8,16 & 7,85 & 8,48 \\
\hline Rwanda & 15,86 & 16,99 & 13,23 \\
\hline Samoa & 45,76 & 41,97 & 45,61 \\
\hline San Vicente y las Granadinas & 33,70 & 33,04 & 33,40 \\
\hline Santa Lucía & 45,87 & 45,15 & 45,24 \\
\hline Santo Tomé y Príncipe & 18,75 & 20,06 & 16,27 \\
\hline Senegal & 16,76 & 17,02 & \\
\hline Serbia & 19,07 & 18,77 & 20,35 \\
\hline Seychelles & 72,63 & 68,22 & 62,18 \\
\hline Sierra Leona & 11,96 & 19,93 & 18,55 \\
\hline Singapur & 84,20 & 84,52 & 82,87 \\
\hline Sri Lanka & 11,27 & 11,99 & 13,88 \\
\hline Sudáfrica & 8,90 & 8,55 & 8,59 \\
\hline Sudán & 3,92 & 4,44 & 5,04 \\
\hline Suecia & 22,00 & 21,87 & 22,26 \\
\hline Suiza & 19,31 & 18,92 & 19,54 \\
\hline Suriname & 11,46 & 17,49 & 15,64 \\
\hline Swazilandia & 23,86 & 28,33 & 26,41 \\
\hline Tailandia & 24,88 & 27,11 & 28,07 \\
\hline Tanzanía & 17,17 & 18,88 & 17,67 \\
\hline Tayikistán & 16,90 & 18,94 & 22,38 \\
\hline Timor-Leste & 118,93 & 138,67 & 78,26 \\
\hline Tonga & 25,40 & 30,58 & 31,93 \\
\hline Túnez & 20,13 & 16,97 & 18,29 \\
\hline Turquía & 7,70 & 7,99 & 8,15 \\
\hline Ucrania & 22,75 & 21,20 & 20,77 \\
\hline Uganda & 19,38 & 27,18 & 22,81 \\
\hline Uruguay & 10,85 & 11,91 & 11,41 \\
\hline Vanuatu & 57,14 & 54,84 & 59,44 \\
\hline Venezuela & 3,80 & 5,60 & 5,35 \\
\hline Viet Nam & 14,99 & 15,30 & 14,20 \\
\hline Zambia & 7,34 & 7,70 & 8,34 \\
\hline
\end{tabular}

Fuente: Doing Business, Banco Mundial 
Tabla 3. Comercio de Mercaderías como porción del PIB (MERC)

\begin{tabular}{|c|c|c|c|}
\hline ECONOMÍA & MERC 2010 & MERC 2011 & MERC 2012 \\
\hline Afganistán & 34,78 & 37,86 & 31,94 \\
\hline Albania & 49,89 & 56,99 & 55,48 \\
\hline Alemania & 70,02 & 75,21 & 75,15 \\
\hline Angola & 81,56 & 83,74 & 84,10 \\
\hline Antigua y Barbuda & 48,17 & 46,78 & 48,99 \\
\hline Arabia Saudita & 67,96 & 74,14 & 72,22 \\
\hline Argelia & 60,50 & 60,65 & 59,11 \\
\hline Argentina & 26,94 & 28,31 & 24,82 \\
\hline Armenia & 51,73 & 54,03 & 57,19 \\
\hline Australia & 36,28 & 37,07 & 33,79 \\
\hline Austria & 82,50 & 88,67 & 87,31 \\
\hline Azerbaiyán & 62,80 & 67,72 & 61,54 \\
\hline Bahamas & 41,75 & 48,25 & 54,61 \\
\hline Bahrein & 105,90 & 111,49 & 116,59 \\
\hline Bangladesh & 46,85 & 54,20 & 51,06 \\
\hline Belarús & 108,96 & 145,96 & 145,24 \\
\hline Bélgica & 170,20 & 183,66 & 182,46 \\
\hline Belice & 71,76 & 81,02 & 95,39 \\
\hline Bhután & 94,31 & 93,78 & 86,51 \\
\hline Bolivia & 59,39 & 66,79 & 70,28 \\
\hline Bosnia y Herzegovina & 83,61 & 92,59 & 90,10 \\
\hline Botswana & 75,29 & 86,02 & 96,28 \\
\hline Brasil & 18,36 & 19,91 & 21,16 \\
\hline Bulgaria & 96,68 & 113,54 & 115,83 \\
\hline Burundi & 30,10 & 37,18 & 36,81 \\
\hline Cabo Verde & 47,28 & 54,49 & 46,60 \\
\hline Camboya & 106,15 & 126,66 & 136,61 \\
\hline Camerún & 40,06 & 43,55 & 43,82 \\
\hline Canadá & 48,96 & 51,47 & 51,04 \\
\hline Chile & 59,90 & 62,24 & 59,37 \\
\hline China & 50,15 & 49,74 & 46,99 \\
\hline Chipre & 43,11 & 42,23 & 39,76 \\
\hline Colombia & 28,05 & 33,42 & 32,21 \\
\hline Congo, República Democrática del & 47,75 & 50,77 & 45,17 \\
\hline Corea, República de & 81,46 & 89,78 & 87,30 \\
\hline Costa Rica & 63,41 & 64,57 & 63,82 \\
\hline Croacia & 54,12 & 58,64 & 58,95 \\
\hline Dinamarca & 58,24 & 62,18 & 63,18 \\
\hline Djibouti & 40,67 & 48,69 & 49,87 \\
\hline Dominica & 53,61 & 50,16 & 47,42 \\
\hline Ecuador & 56,40 & 60,71 & 58,46 \\
\hline Egipto, República Árabe de & 36,26 & 37,89 & 37,75 \\
\hline El Salvador & 60,30 & 66,00 & 65,55 \\
\hline República Eslovaca & 148,94 & 166,54 & 174,33 \\
\hline Eslovenia & 126,40 & 139,87 & 141,03 \\
\hline España & 41,99 & 46,97 & 47,22 \\
\hline Estados Unidos & 21,71 & 24,12 & 23,90 \\
\hline Estonia & 125,35 & 152,42 & 151,03 \\
\hline Etiopía & 37,20 & 37,53 & 35,04 \\
\hline
\end{tabular}




\section{Continuación tabla 3}

\begin{tabular}{|c|c|c|c|}
\hline ECONOMÍA & MERC 2010 & MERC 2011 & MERC 2012 \\
\hline Fiji & 82,16 & 86,32 & 84,01 \\
\hline Filipinas & 55,09 & 49,98 & 46,91 \\
\hline Finlandia & 58,44 & 62,28 & 60,21 \\
\hline Francia & 44,18 & 47,32 & 47,59 \\
\hline Gambia & 33,62 & 48,81 & 52,51 \\
\hline Georgia & 59,58 & 64,06 & 64,49 \\
\hline Ghana & 58,69 & 72,67 & 71,87 \\
\hline Granada & 44,31 & 46,04 & 46,16 \\
\hline Grecia & 29,06 & 31,93 & 38,03 \\
\hline Guatemala & 53,95 & 56,69 & 53,82 \\
\hline Guinea & 60,73 & 69,83 & 65,47 \\
\hline Guyana & 100,78 & 112,06 & 112,24 \\
\hline Haití & 56,25 & 50,37 & 45,88 \\
\hline Honduras & 94,81 & 106,12 & 102,89 \\
\hline $\begin{array}{l}\text { Hong Kong, } \\
\text { Región Administrativa Especial }\end{array}$ & 368,30 & 388,88 & 398,88 \\
\hline Hungría & 144,04 & 156,24 & 159,76 \\
\hline India & 33,75 & 40,82 & 42,10 \\
\hline Indonesia & 41,37 & 44,56 & 43,16 \\
\hline Iraq & 67,50 & 69,20 & 70,15 \\
\hline Irlanda & 84,42 & 85,10 & 85,13 \\
\hline Islandia & 67,84 & 72,56 & 72,35 \\
\hline Islas Salomón & 92,15 & 101,50 & 97,00 \\
\hline Israel & 51,63 & 55,62 & 53,84 \\
\hline Italia & 45,46 & 49,27 & 48,98 \\
\hline Jamaica & 49,53 & 57,08 & 56,10 \\
\hline Japón & 26,64 & 28,42 & 28,37 \\
\hline Jordania & 85,49 & 93,40 & 92,14 \\
\hline Kazajstán & 61,52 & 66,29 & 67,23 \\
\hline Kenya & 53,21 & 59,85 & 55,67 \\
\hline Kuwait & 77,25 & 80,38 & 80,23 \\
\hline Lesotho & 146,05 & 147,63 & 158,92 \\
\hline Líbano & 61,78 & 65,90 & 63,79 \\
\hline Lituania & 121,61 & 139,54 & 145,56 \\
\hline Luxemburgo & 86,14 & 88,23 & 84,76 \\
\hline Malasia & 146,74 & 143,66 & 139,00 \\
\hline Malawi & 60,00 & 68,46 & 86,07 \\
\hline Maldivas & 60,37 & 83,76 & 88,43 \\
\hline Marruecos & 58,56 & 66,34 & 68,25 \\
\hline Mauricio & 68,40 & 68,55 & 68,61 \\
\hline México & 57,86 & 60,73 & 63,33 \\
\hline Mongolia & 99,63 & 130,30 & 107,77 \\
\hline Montenegro & 63,64 & 70,55 & 69,43 \\
\hline Mozambique & 81,95 & 78,98 & 75,82 \\
\hline Nepal & 37,45 & 35,51 & 38,84 \\
\hline Nicaragua & 67,40 & 75,44 & 80,12 \\
\hline Nigeria & 35,00 & 41,23 & 35,90 \\
\hline Noruega & 49,41 & 50,96 & 49,41 \\
\hline Nueva Zelandia & 43,22 & 45,60 & 44,07 \\
\hline
\end{tabular}




\section{Continuación tabla 3}

\begin{tabular}{|c|c|c|c|}
\hline ECONOMÍA & MERC 2010 & MERC 2011 & MERC 2012 \\
\hline Omán & 96,19 & 101,40 & 103,46 \\
\hline Países Bajos & 140,34 & 152,04 & 161,87 \\
\hline Pakistán & 33,42 & 32,47 & 30,57 \\
\hline Panamá & 96,22 & 109,28 & 103,80 \\
\hline Papua Nueva Guinea & 102,23 & 94,47 & 76,66 \\
\hline Paraguay & 82,60 & 80,09 & 76,21 \\
\hline Perú & 44,23 & 49,41 & 45,78 \\
\hline Polonia & 71,90 & 77,42 & 77,41 \\
\hline Portugal & 54,32 & 59,75 & 61,39 \\
\hline Reino Unido & 43,87 & 47,77 & 46,66 \\
\hline República Checa & 130,80 & 145,82 & 151,27 \\
\hline República Dominicana & 43,63 & 46,99 & 45,15 \\
\hline Rumania & 67,74 & 76,39 & 75,49 \\
\hline Federación de Rusia & 42,58 & 44,41 & 42,86 \\
\hline Rwanda & 30,73 & 34,96 & 34,63 \\
\hline Samoa & 64,46 & 63,36 & 61,62 \\
\hline San Vicente y las Granadinas & 55,68 & 54,67 & 56,74 \\
\hline Santa Lucía & 70,04 & 66,45 & 67,52 \\
\hline Santo Tomé y Príncipe & 61,21 & 57,38 & 57,34 \\
\hline Senegal & 53,69 & 58,52 & 63,72 \\
\hline Serbia & 71,72 & 72,32 & 79,68 \\
\hline Seychelles & 107,90 & 116,37 & 125,62 \\
\hline Sierra Leona & 43,10 & 70,17 & 63,37 \\
\hline Singapur & 280,29 & 282,88 & 274,69 \\
\hline Sri Lanka & 44,61 & 51,55 & 48,10 \\
\hline Sudáfrica & 47,95 & 54,38 & 54,93 \\
\hline Sudán & 32,68 & 28,11 & 19,36 \\
\hline Suecia & 66,40 & 67,84 & 63,87 \\
\hline Suiza & 67,73 & 67,24 & 67,09 \\
\hline Suriname & 78,37 & 94,07 & 83,80 \\
\hline Swazilandia & 96,62 & 93,11 & 95,07 \\
\hline Tailandia & 117,97 & 130,58 & 130,37 \\
\hline Tanzanía & 50,54 & 63,48 & 58,81 \\
\hline Tayikistán & 68,28 & 68,42 & 67,29 \\
\hline Timor-Leste & 33,69 & 31,20 & 28,19 \\
\hline Tonga & 45,24 & 49,01 & 47,92 \\
\hline Túnez & 87,71 & 90,96 & 91,64 \\
\hline Turquía & 40,95 & 48,50 & 49,32 \\
\hline Ucrania & 82,38 & 92,43 & 86,73 \\
\hline Uganda & 39,19 & 50,28 & 41,55 \\
\hline Uruguay & 39,47 & 39,46 & 40,71 \\
\hline Vanuatu & 47,64 & 47,30 & 44,48 \\
\hline Venezuela & 26,73 & 44,56 & 41,27 \\
\hline Viet Nam & 135,49 & 150,26 & 146,56 \\
\hline Zambia & 77,34 & 84,26 & 80,35 \\
\hline
\end{tabular}

Fuente: Doing Business, Banco Mundial 
Para la estadística descriptiva se utilizaron medias, desviaciones estándar, histogramas y gráficos de dispersión. Se utilizó la prueba de $t$ de Student de muestras pareadas para evaluar la variación de los promedios de cada variable entre el inicio y el final del período analizado. Con la prueba de KolmogorovSmirnov (KS) se verificó la normalidad de la distribución de las variables. Las variables que no tienen distribución normal se transformaron usando la función logaritmo base 10 para normalizarlas. Para analizar la evolución de las variaciones del presente estudio en el tiempo, se calcularon las variaciones anuales de las variables DF LOGSERV y LOGMERC (DIF_DF, DIF_LOGSERV y DIF_LOGMERC respectivamente). Se utilizó la correlación de Pearson (Chatterjee, 1986., 379-416) para evaluar la asociación entre las variables. Se construyeron modelos de regresión lineal por mínimos cuadrados para evaluar el peso de las variables LOGSERV y LOGMERC para estimar la DF. Los análisis estadísticos se realizaron en Excel y Matlab.

\section{Resultados}

En la tabla 4 se presenta el promedio y desviación estándar (STD) de las variables DF, SERV y MERC para el período 2010 a 2012 y para 141 economías del mundo.

Tabla 4. Estadística descriptiva de las variables DF, SERV y MERC

\begin{tabular}{l|c|c|c|c|c|c|c|c|c}
\hline & DF & DF & DF & SERV & SERV & SERV & MERC & MERC & MERC \\
& $\mathbf{2 0 1 0}$ & $\mathbf{2 0 1 1}$ & $\mathbf{2 0 1 2}$ & $\mathbf{2 0 1 0}$ & $\mathbf{2 0 1 1}$ & $\mathbf{2 0 1 2}$ & $\mathbf{2 0 1 0}$ & $\mathbf{2 0 1 1}$ & $\mathbf{2 0 1 2}$ \\
\hline Promedio & 61,5 & 62,2 & 63,0 & 24,7 & 25,3 & 25,3 & 69,8 & 75,6 & 75,0 \\
\hline STD & 12,2 & 12,2 & 11,9 & 24,2 & 25,3 & 24,6 & 43,3 & 46,2 & 47,2 \\
\hline
\end{tabular}

Fuente: elaborado por los autores

Con la prueba de $t$ de Student para muestras pareadas se encontró un crecimiento para DF de $2.4 \%(p<0.0001)$ y para LOGMERC $7.5 \%$ $(p<0.0001)$, en tanto que LOGSERV tuvo un crecimiento de $2.4 \%$ ( $p>0.05)$.

En los siguientes histogramas (figura 1), las barras corresponden a la distribución de las variables de análisis, y la línea corresponde a una serie aleatoria con media y desviación estándar iguales a la media y desviación estándar de la variable analizada. En el panel superior se observa que la variable DF se ajusta a la distribución normal, a diferencia de las variables SERV y MERC que tienen una distribución asimétrica y leptokúrtica. En el panel inferior se observa que la transformación logarítmica de estas dos variables se ajusta a la distribución normal.

En la tabla 5 se presentan los resultados de la prueba KS, con lo que se encontró que la variable DF y las variables con transformación logarítmicas tienen distribución normal para el período del estudio, en tanto que las variables sin transformación logarítmica no tienen distribución normal.

En los gráficos de dispersión de la figura 2 se observa una correlación positiva de la variable DF con las variables LOGSERV y LOGMERC. También se muestra una correlación positiva entre LOGSERV y LOGMERC.

Para analizar la variación año tras año de las variables DF, LOGSERV y LOGMERC, se definieron las variables DIF_DF, DIF_ LOGSERV y DIF_LOGMERC, con las cuales se evidencia una mayor correlación entre la DF con la actividad comercial de mercaderías que entre la DF y la actividad comercial de servicios y una muy superior entre la actividad comercial de mercaderías con la actividad comercial de servicios (tabla 5). 


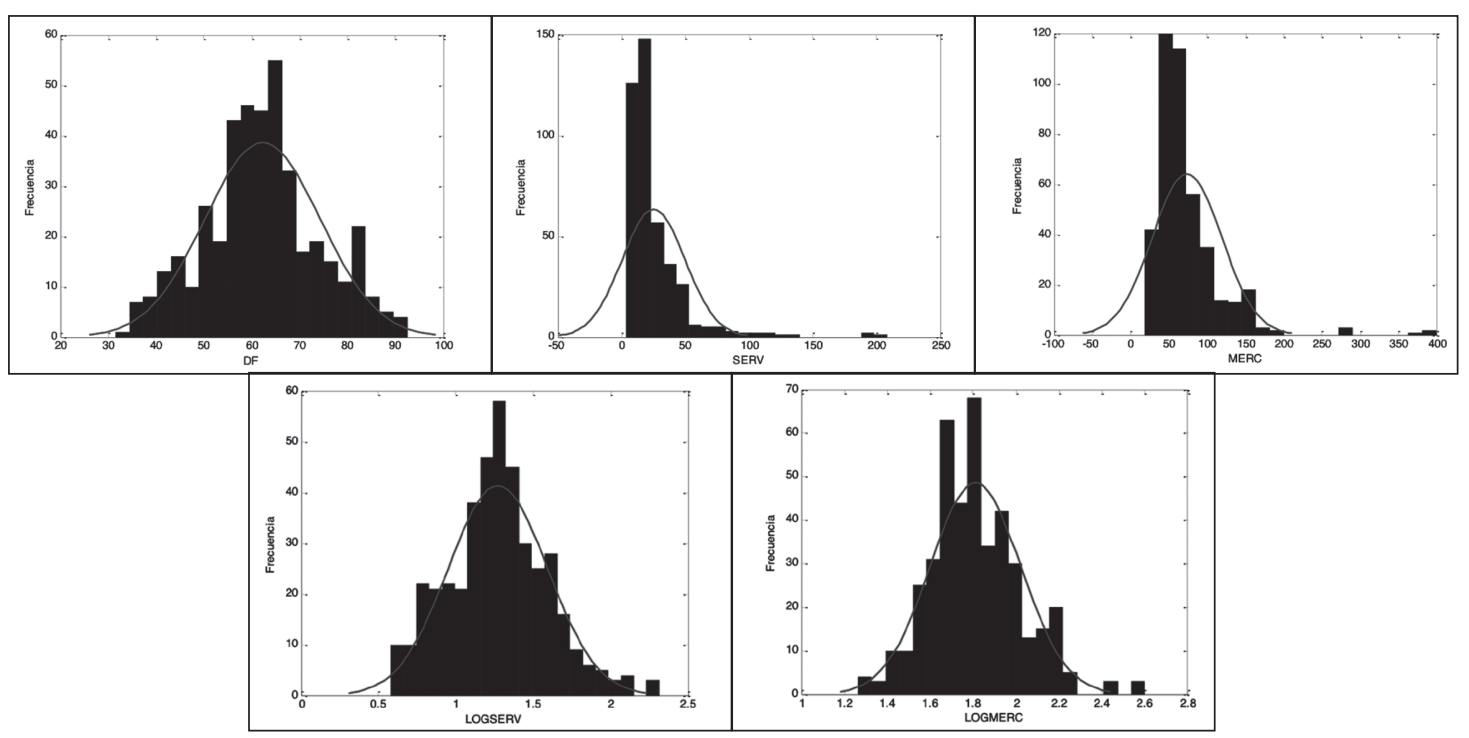

Figura 1. Histogramas de frecuencia de DF, SERV, MERC, LOGSERV y LOGMERC.

Fuente: elaborado por los autores

Tabla 5. Prueba de Kolmogorov-Smirnov (KS)

\begin{tabular}{l|c|c|c}
\hline \multicolumn{1}{c|}{ VARIABLE } & KS & $\mathbf{P}$ & DISTRIBUCIÓN NORMAL \\
\hline DF 2010 & 0.0567 & 0.9733 & SÍ \\
\hline DF 2011 & 0.0496 & 0.9939 & SÍ \\
\hline DF 2012 & 0.0638 & 0.9283 & SÍ \\
\hline SERV 2010 & 0.2270 & 0.0011 & NO \\
\hline SERV 2011 & 0.2199 & 0.0018 & NO \\
\hline SERV 2012 & 0.2128 & 0.0028 & NO \\
\hline MERC 2010 & 0.1844 & 0.0142 & NO \\
\hline MERC 2011 & 0.1773 & 0.0206 & NO \\
\hline MERC 2012 & 0.1702 & 0.0295 & NO \\
\hline LOGSERV 2010 & 0.0496 & 0.9939 & SÍ \\
\hline LOGSERV 2011 & 0.0496 & 0.9939 & SÍ \\
\hline LOGSERV 2012 & 0.0426 & 0.9994 & Sí \\
\hline LOGMERC 2010 & 0.0567 & 0.9733 & SÍ \\
\hline LOGMERC 2011 & 0.0709 & 0.8577 & SÍ \\
\hline LOGMERC 2012 & 0.0709 & 0.8577 & Sí \\
\hline
\end{tabular}

Fuente: elaborado por los autores

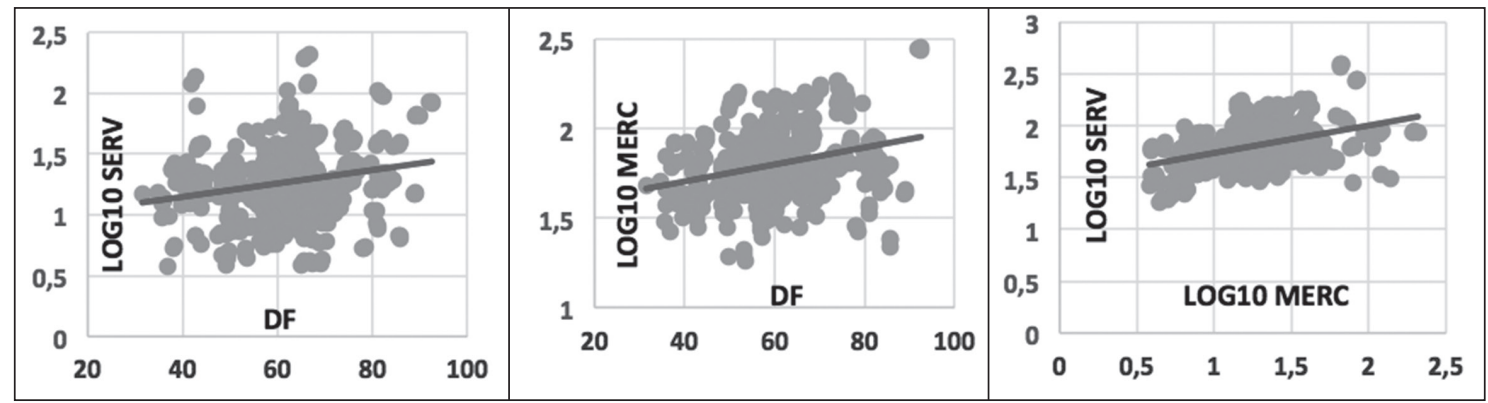

Figura 2. Gráficos de dispersión. En el panel izquierdo DF Vs. LOGSERV, en el panel central DF Vs. LOGMERC y en el panel derecho LOGMERC Vs. LOGSERV. Fuente: elaborado por los autores 
Tabla 6. Coeficientes de Pearson entre las diferencias anuales de DF (DIF_DF), diferencias anuales de LOGSERV (DIF_LOGSERV) y diferencias anuales de LOGMERC (DIF_LOGMERC)

\begin{tabular}{l|c|c|c}
\hline & DIF_DF & DIF_LOGSERV & DIF_LOGMERC \\
\hline DIF_DF & 1 & 0,0614 & 0,1137 \\
\hline DIF_LOGSERV & 0,0614 & 1 & 0,3188 \\
\hline DIF_LOGMERC & 0,1137 & 0,3188 & 1 \\
\hline
\end{tabular}

Fuente: elaborado por los autores

Con el modelo construido usando regresión lineal múltiple por mínimos cuadrados (Ecuación 1), se obtuvo un $\mathrm{R}^{2}$ de 0,086 $(p=0,021)$ y residuales con distribución normal (figura 3):

Ecuación 1: $D F=332,19+44,14^{*}$ LOGSERV+12 9,3*LOGMERC

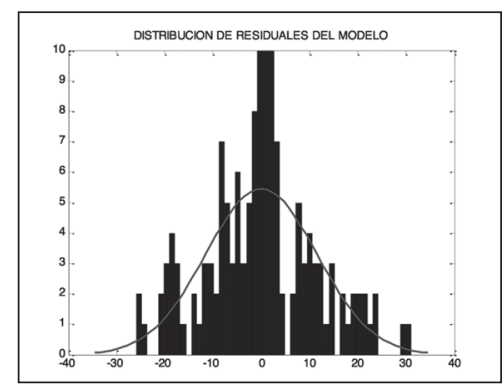

Figura 3. Distribución de residuales del modelo de regresión Fuente: elaborado por los autores

Se encontró que la variable LOGMERC tiene un peso mucho mayor que la variable LOGSERV para explicar la tendencia de DF. En La tabla 6 se muestran los coeficientes de las variables y los intervalos de confianza inferior (IC INF) y superior (IC SUP).

Tabla 6. Parámetros del modelo de regresión

\begin{tabular}{l|c|c|c}
\hline \multicolumn{1}{c|}{ VARIABLE } & COEFICIENTE & IC INF & IC SUP \\
\hline Independiente & 332,19 & 16,27 & 50,17 \\
\hline LOGSERV & 44,14 & $-2,22$ & 11,05 \\
\hline LOGMERC & 129,3 & 2,76 & 23,1 \\
\hline
\end{tabular}

Fuente: elaborado por los autores

\section{Discusión}

El crecimiento de la DF en el período 20102012 indica que existe un acatamiento significativo por parte de las economías a las políticas de desregulación planteadas por el BM en el transcurso del mismo período. Sin embargo, las evidencias encontradas muestran que el impacto de estas medidas y la DF se asocian con el incremento importante en el comercio de mercaderías (LOGMERC), en tanto que el crecimiento del comercio de servicios (LOGSERV) no fue estadísticamente significativo.

El crecimiento encontrado en la DF de 2,4\% es de magnitud importante dado que es una variable que mide solo una fracción de lo que 
conlleva a los negocios de las economías a un mejor desempeño 5 .

Por el contrario, el crecimiento de LOGSERV de $2,4 \%$ no mostró significancia estadística en este estudio y, además, su magnitud fue cerca de la tercera parte del crecimiento de LOGMERC que aumentó en 7,5\%.

El análisis de correlaciones también refleja que la variación de la DF (DIF_DF) tiene una mayor asociación con la variación de la actividad comercial de mercaderías (DIF_LOGMERC) que con la variación la actividād comercial de servicios (DIF LOGSERV), $\quad r=0,1137$ y $r=0,0614$, respectivamente, lo cual es coherente con los demás resultados del estudio para indicar que el efecto de la desregulación afecta más la actividad en mercaderías que la actividad en servicios.

A pesar estas diferencias planteadas en las correlaciones, llama la atención que hay una correlación relativamente importante $(r=0,3188)$ entre la dif_logmerc y dif_logserv. Esto lleva a considerar que la actividad de servicios es un fenómeno derivado en mayor grado del comercio de mercaderías que de los procesos de desregulación.

El modelo de regresión construido en el presente estudio no tiene propósitos predictivos o de extrapolación. La intención con este modelo es evaluar el peso que tienen las variables logmerc y logserv para explicar el comportamiento de DF. Los coeficientes obtenidos muestran que logmerc es suficiente para explicar el comportamiento de DF como variable dependiente.

Con todas las técnicas estadísticas se llega a las mismas conclusiones sobre la relación que hay entre las variables del estudio.

\section{Conclusiones}

Los resultados respaldan lo que los autores plantean en su hipótesis $\mathrm{y}$, adicionalmente, dan a conocer que, de manera más crítica que lo planteado allí en la que se esbozan preocupaciones por el escaso papel de políticas de desregulación en el comercio de servicios a la hora de diseñar el instrumento DF, las desregulaciones planteadas por el Banco Mundial para el mejoramiento de las economías en sus sectores de servicios y mercaderías tienen un efecto solamente en el sector de mercaderías y no en el de servicios.

Con toda la evidencia hallada se entiende que difícilmente se puedan lograr en el mundo, mediante la implementación de políticas de desregulación trazadas por el BM que mide la DF de cada país, economías que se consoliden en el negocio de los servicios por encima del de mercaderías.

Las medidas que se estimulan para mejorar posiciones con respecto a la DF y que inducen la desregulación, en el sector de mercaderías, tienen como consecuencia hacer aún más profundas las diferencias entre quienes obtienen de manera autónoma, regidos por sus propias políticas económicas, mayores beneficios y productividad económica por su participación en el negocio de intangibles, y quienes se rigen por los lineamientos del BM terminando por alcanzar los réditos marginales inherentes al negocio de bienes materiales ${ }^{6}$.

Si bien el BM deja en claro las limitaciones del indicador DF es importante señalar que no se han descrito aspectos ni se han incluido para el diseño de este, gestiones que orienten la desregulación y que desencadenen y posibiliten un mejor desarrollo en la actividad de servicios como se puede entender en la observación de la forma como están planteadas las acciones orientadas a la desregulación para incrementar la facilidad en los negocios hasta el momento ${ }^{7}$. Por el contrario se excluyen algunas del comercio en general que tienen consecuencias favorecedoras para el comercio de servicios, tales como los

5 "Las clasificaciones no consideran muchos otros factores que influyen en el éxito del negocio, tales como la proximidad a los grandes mercados, la infraestructura, la seguridad, las condiciones económicas, y la transparencia en la contratación pública. DB cubre solo el sector formal" (McKay \& Schreiner, 2013).

6 Para una mayor comprensión sobre la riqueza proveniente de actividades materiales vs. actividades intangibles, véase Drucker (1993).

7 Global excluyendo obtención de electricidad, Apertura de un negocio, Manejo de permisos de construcción, Obtención de electricidad, Registro de propiedades, Obtención de crédito, Protección de los inversionistas minoritarios, Pago de impuestos, Comercio transfronterizo, Cumplimiento de contratos y Resolución de la insolvencia. 
relacionados con la infraestructura tecnológica digital, la flexibilización del sistema laboral que rige el empleo en la producción y comercio de intangibles, el fortalecimiento del sistema institucional que regula la actividad de los productos inmateriales, las medidas que mejoran la solución a los problemas de la innovación y la tecnología o los aspectos relacionados con educación y entrenamiento:

There are many other things which we don't capture in the Doing Business report, and we're really quite open and clear about the limitations. We have nothing to say on education and training. We don't really cover aspects of microeconomic stability. We don't really look at issues of innovation and technology and so on. However, the indicators that we cover do capture very important dimensions of the business environment. It does matter a great deal for the business community whether, for instance, you have a tax administration system that is very burdensome and very complicated, or whether it is light and simple and transparent. In fact, over the years, in designing the indicators we have used two primary sources of insight for, essentially, capturing these dimensions of the business environment (the center for strategic and international, 2013).

Por todo lo anterior podemos concluir que el indicador DF, tal y como hasta la fecha lo ha definido el BM, está orientado únicamente a medir la flexibilización del comercio de mercaderías, y sus políticas para que las economías exhiban un mejor desempeño en la DF contribuyen al incremento de la actividad en el sector. Por el contrario, no mide la flexibilización del comercio de servicios, y las mejoras que las economías presentan en este sector son únicamente el resultado de actividades accesorias derivadas del comercio de mercaderías, mas no como una consecuencia de las políticas de desregulación propuestas por el BM que mide el indicador DF.

\section{Recomendaciones}

Es importante avanzar en el estudio acá desarrollado abarcando un mayor número de períodos o efectuando una caracterización de los mismos de acuerdo con las dinámicas socio-políticas de los países, o mejor aún, estableciendo una caracterización del estudio de acuerdo con la naturaleza de su actividad económica, dado que el costo de la pretendida universalidad del instrumento DF ha sido la pérdida de resolución del mismo como mecanismo para revisar las particularidades de las economías y ajustarse a sus dificultades para plantear políticas adecuadas a las necesidades propias, derivadas de una trayectoria comercial, ubicación geográfica o aspectos culturales que explican, entre otros, el grado de informalidad de sus sectores comerciales.

Es importante hacer estudios que cubran un mayor número de economías y de períodos para el cálculo de la $\mathrm{DF}^{8}$, teniendo en cuenta siempre que el BM ha modificado sus metodologías para algunos períodos.

Se recomienda una revisión del instrumento desarrollado por el BM para que incluya nuevas consideraciones que permitan desregular el sector del comercio de servicios.

\section{Referencias bibliográficas}

- Banco Mundial, G. (2014). Comercio de mercaderías. Obtenido de http://datos.bancomundial.org/ indicador/TG.VAL.TOTL.GD.ZS/ countries $/ 1 \mathrm{~W}$ ?display=default.

- Banco Mundial, G. (2014). Doing Business 2015. Going Beyond Efficiency. Recuperado el 12 de 11 de 2014, de http:// espanol.doingbusiness.org/data/ /media/ GIAWB/Doing\%20Business/Documents/ Annual-Reports/English/DB15-Chapters/ DB15-DTF-and-DBRanking.pdf.

- Banco Mundial, G. (2014). Doing Business. Midiendo regulaciones para hacer negocios. Recuperado el 15 de 9 de 2014, de http://espanol.doingbusiness.org/data/ distance-to-frontier

8 Las metodologías para el cálculo de la DF ha cambiado en algunos períodos. 
- Banco Mundial. (2014). Comercio de servicios. Comercio de Servicios. Recuperado el 15 de 10 de 2014, de http://datos.bancomundial.org/indicador/BG.GSR.NFSV. GD.ZS/countries/1W?display=default

- Chatterjee, S. a. ( 1986.). Influential Observations, High Leverage Points, and Outliers in Linear Regression. Statistical Science.

- Drucker, P. F. (1993). La sociedad poscapitalista. Bogotá: Norma.

- McKay, P. Z., \& Schreiner, T. O. (2013). Reference \& User Services Quarterly,. Best of the Best Business Websites (Free Resources), 2(53), 188-190. Obtenido de http://web.a.ebscohost.com/ehost/ pdfviewer/pdfviewer?sid=fe687511-0f9c4063-acc9-3a57d65c49b7\%40sessionmgr 4004\&vid=1\&hid $=4207$
- Plata, L. C. (2005). Valoración de activos intangibles, la nueva riqueza de las empresas. Revista de derecho(24), 140155. Recuperado el 6 de 10 de 2014, de http://web.b.ebscohost.com/ehost/ pdfviewer/pdfviewer?sid=4af69d1d-372543fd-aafd-d93cef477c9d\%40sessionmgr1 $14 \&$ vid $=6 \&$ hid $=116$

- The Center For Strategic And International, S. (2013). The Center For Strategic And International Studies Holds A Discussion On The World Bank's Doing Business 2014 Report. Financial Markets Regulatory Wire. Obtenido de http://web.a.ebscohost.com/ ehost/detail/detail?sid=416c7097-109349b1-9ec5-a85171026a8f\%40sessionmgr 4004\&vid=1\&hid $=4207 \&$ bdata $=J \mathrm{mxhbmc9}$ ZXMmc2I0ZT1laG9zdC1saXZl\#db=buh\&A $\mathrm{N}=8 \mathrm{VU} 82361919980$ 